\title{
Pharmacological reduction of adult hippocampal neurogenesis modifies functional brain circuits in mice exposed to a cocaine conditioned place preference paradigm
}

\author{
Estela Castilla-Ortega', Eduardo Blanco ${ }^{2}$, Antonia Serrano', \\ David Ladrón de Guevara-Miranda ${ }^{3}$, María Pedraz', Guillermo Estivill-Torrús ${ }^{4}$, \\ Francisco Javier Pavón', Fernando Rodríguez de Fonseca' \& Luis J. Santín ${ }^{3}$ \\ Unidad de Gestión Clínica de Salud Mental, Instituto de Investigación Biomédica de Málaga (IBIMA), Hospital Regional Universitario de Málaga, Spain', Departament \\ de Pedagogia i Psicología, Facultat d'Educació, Psicologia i Treball Social, Universitat de Lleida, Spain², Departamento de Psicobiología y Metodología de las Ciencias \\ del Comportamiento, Instituto de Investigación Biomédica de Málaga (IBIMA), Facultad de Psicología, Universidad de Málaga, Spain ${ }^{3}$ and Unidad de Gestión Clínica \\ Intercentros de Neurociencias y ECAI de Microscopía, Instituto de Investigación Biomédica de Málaga (IBIMA), Hospitales Universitarios Regional de Málaga y \\ Virgen de la Victoria, Spain ${ }^{4}$
}

\begin{abstract}
We investigated the role of adult hippocampal neurogenesis in cocaine-induced conditioned place preference (CPP) behaviour and the functional brain circuitry involved. Adult hippocampal neurogenesis was pharmacologically reduced with temozolomide (TMZ), and mice were tested for cocaine-induced CPP to study c-Fos expression in the hippocampus and in extrahippocampal addiction-related areas. Correlational and multivariate analysis revealed that, under normal conditions, the hippocampus showed widespread functional connectivity with other brain areas and strongly contributed to the functional brain module associated with CPP expression. However, the neurogenesisreduced mice showed normal CPP acquisition but engaged an alternate brain circuit where the functional connectivity of the dentate gyrus was notably reduced and other areas (the medial prefrontal cortex, accumbens and paraventricular hypothalamic nucleus) were recruited instead of the hippocampus. A second experiment unveiled that mice acquiring the cocaine-induced CPP under neurogenesis-reduced conditions were delayed in extinguishing their drug-seeking behaviour. But if the inhibited neurons were generated after CPP acquisition, extinction was not affected but an enhanced long-term CPP retention was found, suggesting that some roles of the adult-born neurons may differ depending on whether they are generated before or after drug-contextual associations are established. Importantly, cocaine-induced reinstatement of CPP behaviour was increased in the TMZ mice, regardless of the time of neurogenesis inhibition. The results show that adult hippocampal neurogenesis sculpts the addiction-related functional brain circuits, and reduction of the adult-born hippocampal neurons increases cocaine seeking in the CPP model.
\end{abstract}

Keywords Brain networks connectivity, drug addiction vulnerability, early immediate gene c-Fos, extinction and reinstatement, principal components factorial analysis PCA, temozolomide TMZ.

Correspondence to: Luis J. Santín, Departamento de Psicobiología y Metodología de las CC, Facultad de Psicología, Universidad de Málaga, Campus de Teatinos S/N, Málaga 29071, Spain. E-mail: luis@uma.es

\section{INTRODUCTION}

Drug addiction is a chronic disorder of the nervous system characterized by compulsive drug seeking and taking with repeated attempts at abstinence and recurrent relapse, carrying many health and socio-economic implications (Cleva \& Gass 2010; Karila et al. 2012). In particular, cocaine abuse constitutes a serious public health problem as cocaine remains the second most commonly used illicit drug worldwide after cannabis (Karila et al. 2012). Drug addiction is considered a disorder of aberrant learning and memory because long-lasting 
associations are formed between a drug's effects and the context where they were experienced, so an individual would be driven to compulsively seek and take the drug when exposed to the environment where the drug was previously used (Hyman 2005; Cleva \& Gass 2010; Torregrossa, Corlett \& Taylor 2011). Therefore, efforts are made to identify the neurobiological mechanisms or vulnerability factors involved in the establishment of the drug-associated memories as well as their extinction, for therapeutic purposes (Sartor \& Aston-Jones 2014).

In this regard, the hippocampus is a key structure for the contextual memories related to drug addiction. It is required to acquire the drug-context relationships (Meyers et al. 2006; Hernandez-Rabaza et al. 2008) to extinguish and recall a previously conditioned association (Cleva \& Gass 2010) and for both contextual and drug-induced reinstatement of cocaine seeking (Fuchs et al. 2007; Rogers \& See 2007). One neuroplastic event within the hippocampus that may support these roles is adult hippocampal neurogenesis. This is suggested because the mechanisms underlying drug addiction may not differ from those involved in other forms of learning (Cleva \& Gass 2010), and the adult-born neurons have been demonstrated to be relevant for hippocampaldependent contextual memory in fear conditioning studies (Castilla-Ortega et al. 2011b). In addition, there is evidence that cocaine and other drugs of abuse downregulate adult hippocampal neurogenesis, which, conversely, is restored by abstinence or the extinction of drug-seeking behaviour (Jun et al. 2012; Deschaux et al. 2014). Following this rationale, it has been proposed that reduced adult hippocampal neurogenesis would constitute a vulnerability factor for establishing and maintaining addiction-related behaviours, while treatments increasing adult-born neurons may have a therapeutic outcome.

Despite the scientific interest generated by this topic (Canales 2007; Jun et al. 2012; Mandyam \& Koob 2012; Chambers 2013; Blanco-Calvo et al. 2014; Geil et al. 2014), there is a lack of studies demonstrating a direct link between adult neurogenesis and drug addiction. While no data are available in humans addicted to drugs, few studies have reduced the number of the adult-born hippocampal neurons to test particular addiction-related phenotypes in rodents. In this regard, the inhibition of adult hippocampal neurogenesis has been demonstrated to enhance cocaine self-administration (Noonan et al. 2010; Deschaux et al. 2014), but experiments have failed to extrapolate these results to the cocaine-induced conditioned place preference paradigm (Brown et al. 2010b; Mustroph et al. 2015). The conditioned place preference (CPP) is a widely used model to assess the reinforcing effects of the drugs (Tzschentke 2007), where drugcontextual associations are established by consistently administering the drug in a specific environment so a drug-seeking animal would prefer the drug-paired context over a non-paired one. It has been reported that neurogenesis-ablated rodents are able to normally acquire a cocaine-induced CPP (Brown et al. 2010b) and to extinguish this behaviour (Mustroph et al. 2015), but this evidence seems insufficient. This is because an important process such as cocaine-primed CPP reinstatement has not been evaluated and, moreover, the hippocampal-cortical-striatal circuits supporting CPP expression in the absence of neurogenesis remain to be assessed (Chambers 2013).

In the present work, adult hippocampal neurogenesis was pharmacologically reduced by the DNA alkylating agent temozolomide (TMZ), and mice were tested in a cocaine-induced CPP paradigm. TMZ is consistently used in adult neurogenesis research and has minimal consequences for animals' health, so mice can readily be tested for behaviour after the TMZ treatment finishes (Garthe, Behr \& Kempermann 2009; Stone et al. 2011; Niibori et al. 2012; Nokia, Anderson \& Shors 2012; Martinez-Canabal et al. 2013; Akers et al. 2014). In addition, we investigated the functional brain circuits underlying CPP expression in control and neurogenesisreduced conditions by analysing c-Fos activity in hippocampal and extrahippocampal addiction-related areas (Peters, Kalivas \& Quirk 2009; Koob \& Volkow 2010). By means of a multivariate statistical approach (Balaban et al. 2014), we unveiled the brain circuits recruited for the expression of cocaine-induced place conditioning. A second experiment aimed to study the involvement of adult-born neurons in the extinction and cocaine-induced reinstatement of drug seeking in the CPP model, which were tested in two independent studies where adult hippocampal neurogenesis was inhibited either before or after the CPP was acquired.

\section{EXPERIMENT I: ROLE OF ADULT HIPPOCAMPAL NEUROGENESIS IN CPP EXPRESSION AND THE UNDERLYING FUNCTIONAL BRAIN CIRCUITS}

Methods

Animals

The animals used for both experiments in this study were male C57BL/6J mice (Janvier, Le Genest-St-Isle, France) that arrived at the animal facility at 11 weeks of age. After 1 week of acclimation, the mice were housed individually in standard laboratory cages including nesting material, and they were maintained on a 12-h light/dark cycle (lights on at 8:00 a.m.) with water and food provided ad libitum. Experimental procedures were performed in accordance with the European (Directive 
2010/63/UE) and Spanish (Real Decreto 53/2013, Ley 32/2007 and 9/2003) regulations of animal research.

\section{TMZ treatment and general health monitoring}

Temozolomide (Sigma, St. Louis, USA) was dissolved in dimethylsulfoxide (DMSO) and then diluted in saline (0.9 percent $\mathrm{NaCl}$ ) to a concentration of 10 percent DMSO. We induced two consecutive cycles of TMZ treatment. In line with previous studies (Garthe et al. 2009; Stone et al. 2011; Niibori et al. 2012; Nokia et al. 2012; Martinez-Canabal et al. 2013; Akers et al. 2014), each cycle consisted of an intraperitoneal (i.p.) dose of 25 mg/kg TMZ (TMZ-mice) or vehicle (VEH-mice) administered once daily for three consecutive days, followed by 4 days without administration (Fig. 1a). Body weight, neurological reflexes, general exploratory and anxietylike behaviour in the hole-board (Castilla-Ortega et al. 2010), and inflammation in the dentate gyrus (DG) were monitored to rule out that the TMZ treatment impaired general health and behaviour (Supporting Information Methods S1).

\section{Bromodeoxyuridine (BrdU) administration}

BrdU is a thymidine analogue that is incorporated into the nuclear DNA of proliferating cells in the S-phase, remaining in the daughter cells so their fate can be studied (Taupin 2007). In each cycle of treatment, BrdU was administered one day following the last TMZ or vehicle injection (days 4 and 11, Fig. 1a). The mice received three daily i.p. doses of $75 \mathrm{mg} / \mathrm{kg}$ BrdU (Sigma) dissolved in saline administered at $3-\mathrm{h}$ intervals (Castilla-Ortega et al. 2014).

\section{Cocaine-induced conditioned place preference}

Behavioural protocols were based on previous reports (Solinas et al. 2008; Ribeiro Do Couto et al. 2009; Brown, Short \& Lawrence 2010a; Bilbao et al. 2013; Poltyrev \& Yaka 2013) and are described in Supporting Information Methods S1 and Fig. 1a. The conditioning phase took place from days 15 to 19 on a group of cocaine-treated mice (cocaine-VEH, $n=23$; cocaine-TMZ, $n=25$ ) that received a daily i.p. administration of cocaine (Sigma, $20 \mathrm{mg} / \mathrm{kg}$ ) paired with one of the compartments of the CPP apparatus and a daily saline i.p. injection paired with the opposite compartment. The groups of salinetreated mice (Sal-VEH, $n=5$; Sal-TMZ, $n=5$ ) received saline in association with both compartments and thus were never exposed to cocaine. The CPP was assessed in a test session on day 22, and drug-seeking behaviour (i.e. preference for the cocaine-paired compartment over the saline-paired one) was calculated as a CPP-score (Poltyrev \& Yaka 2013; Supporting Information Methods S1). (a)
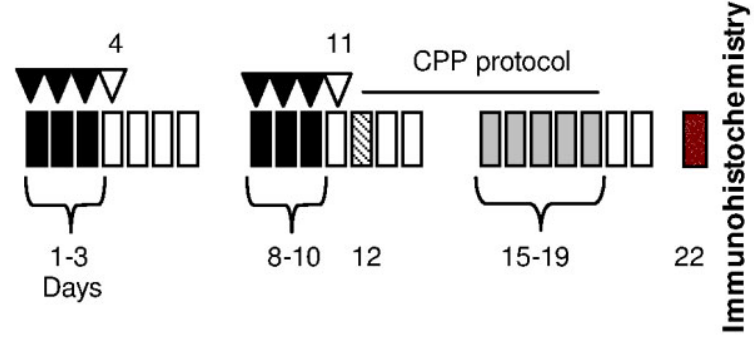

(b)

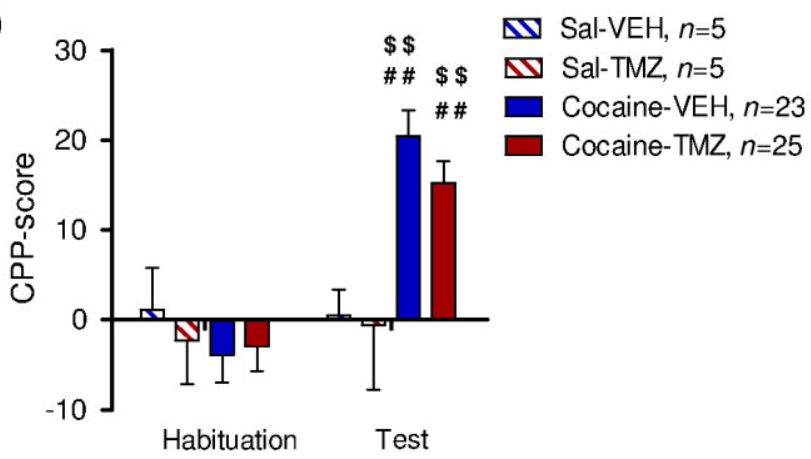

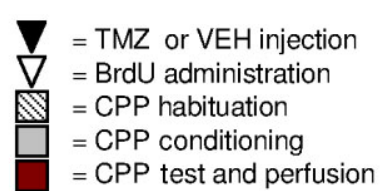

(c)

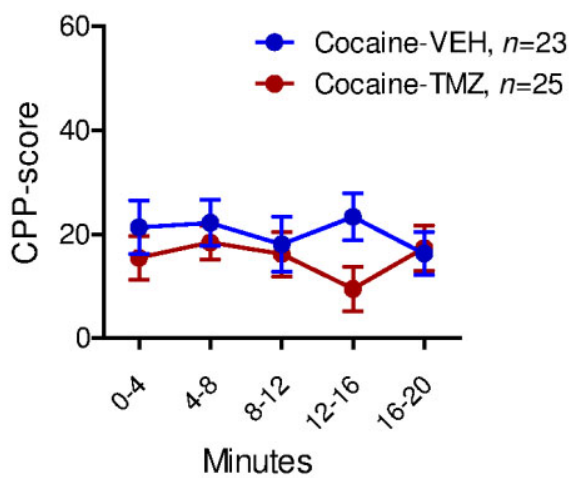

Figure I Effects of the TMZ treatment on CPP conditioning. (a) The protocol used for experiment I. (b) and (c) No differences between the cocaine-VEH and the cocaine-TMZ groups were found in the expression of cocaine-conditioned place preference. Difference versus the Sal group: $\# \# P \leq 0.00$ I; difference from zero: $\$ \$ P \leq 0.001$. Data are expressed as the mean \pm SEM 
Determination of adult hippocampal neurogenesis and c-Fos expression

Animals used for the histological experiments were obtained from the mice that performed the behavioural protocol depicted in Fig. 1. Specifically, the study on the effect of TMZ on adult hippocampal neurogenesis (Fig. 2) and c-Fos expression (Fig. 3) used the salinetreated mice (Sal-VEH, $n=5 ;$ Sal-TMZ, $n=5$ ) and a subset of the cocaine-treated mice that showed a similar CPP-score (CPP-VEH, $n=6$; CPP-TMZ, $n=6$; CPP-scores $\quad$ mean \pm SEM: $\quad \mathrm{CPP}-\mathrm{VEH}=21.55 \pm 1.61$, CPP-TMZ = 23.30 \pm 4.18 ).

The mice were intracardially perfused 90 minutes after the test session. Because both brain hemispheres were equally affected by the TMZ treatment (Supporting Information Fig. S1), adult hippocampal neurogenesis was arbitrarily analysed in the right DG while the left brain hemisphere was used for c-Fos analysis. Immunohistochemical and cell counting procedures are described in our previous work (Castilla-Ortega et al. 2011a, 2012, 2013, 2014) and in Supporting Information Methods S1. The neurogenesis-related markers studied were the proliferating cell nuclear antigen (PCNA) to label cells undergoing proliferation, the microtubuleassociated protein doublecortin (DCX) to label young neurons up to 4 weeks of age, which may have an immature or mature morphology (Brown et al. 2003; Plumpe et al. 2006), and BrdU to label the proliferating population that incorporated BrdU and survived until the time of perfusion. The differentiation of the BrdU+ cells into mature neurons was assessed by double fluorescence immunohistochemistry against BrdU and the mature neuron marker Neuronal Nuclei (NeuN) antigen.

All neurogenesis-related markers were quantified in the suprapyramidal (SupraDG) and infrapyramidal (InfraDG) blades of the DG separately, given their potential differences in their neurogenic dynamics and function (Jinno 2011; Snyder, Ferrante \& Cameron 2012). C-Fos expression associated with the CPP test was analysed in the SupraDG and InfraDG, the hippocampal Cornu ammonis (CA) in the CA3 and CA1 areas, the medial prefrontal cortex ( $\mathrm{MPFC}$ ) in its prelimbic (PrL) and infralimbic (IL) divisions, the accumbens nucleus (Acb) in its core (AcbC) and shell (AcbSh), the dorsomedial $(\mathrm{dmCPU})$ and dorsolateral (dlCPU) striatum, the central (Ce) and basolateral (BLA) amygdala, the medial (MS) and lateral (LS) septum and the paraventricular hypothalamic nucleus (PVN; Supporting Information Fig. S2).

Correlational analysis and description of functional brain circuits

The correlational study (Fig. 4) used 11 of the cocaineVEH and 11 of the cocaine-TMZ mice that performed the behavioural protocol depicted in Fig. 1. Both groups had a similar CPP-score (animals are shown in Fig. 4c) and were processed for immunohistochemistry as described above.

The investigation of the functional brain circuits supporting CPP expression in each experimental condition was performed by means of correlational and multivariate analysis carried out on c-Fos data (Supporting Information Methods S1). This approach has already been employed by previous works to study the functional brain networks underlying a variety of behavioural and physiological processes (Leger et al. 2012; Siette et al. 2013; Balaban et al. 2014; Castilla-Ortega et al. 2014). In short, c-Fos activity among the analysed brain areas was tested for Pearson's correlations, calculating both the $P$ and the $q$ values (Storey 2002) to control for alpha inflation. Significant correlations were graphically represented as a line connecting the two areas, indicating their functional (not necessarily anatomical) connectivity (Siette et al. 2013; Castilla-Ortega et al. 2014). A principal components factorial analysis (PCA) was subsequently performed to reduce the c-Fos data into the functional brain modules underlying behaviour (Balaban et al. 2014). Only a set of brain areas was selected to undergo the PCA so the analysed samples would meet statistical adequacy criteria in both groups (Supporting Information Methods S1).

On the other hand, this same group of animals was used to study the relationship between the neurogenesisrelated markers and the strength of CPP expression by Pearson's correlations (Supporting Information Table S1).

\section{Statistical analysis for comparison of means}

Between- and intra-groups comparisons were carried out with Student's $t$-tests, factorial analyses of variance (ANOVA) or repeated-measures ANOVAs, as appropriate, followed by post hoc Fisher's least significant difference (LSD) analyses when required. A one-sample t-test was used to compare the CPP-scores against zero. Significance was considered at $P \leq 0.05$ in all cases.

\section{Results}

TMZ treatment preserved the general health of the animals

No effects of TMZ administration were found on mice's general health or behaviour besides a delayed body weight gain (as in Nokia et al. 2012) that was rapidly regained before the behavioural testing started. The activated microglia in the DG were similar for both treatments, indicating the absence of inflammatory processes. Data and analysis are shown in Supporting Information Fig. S3. 

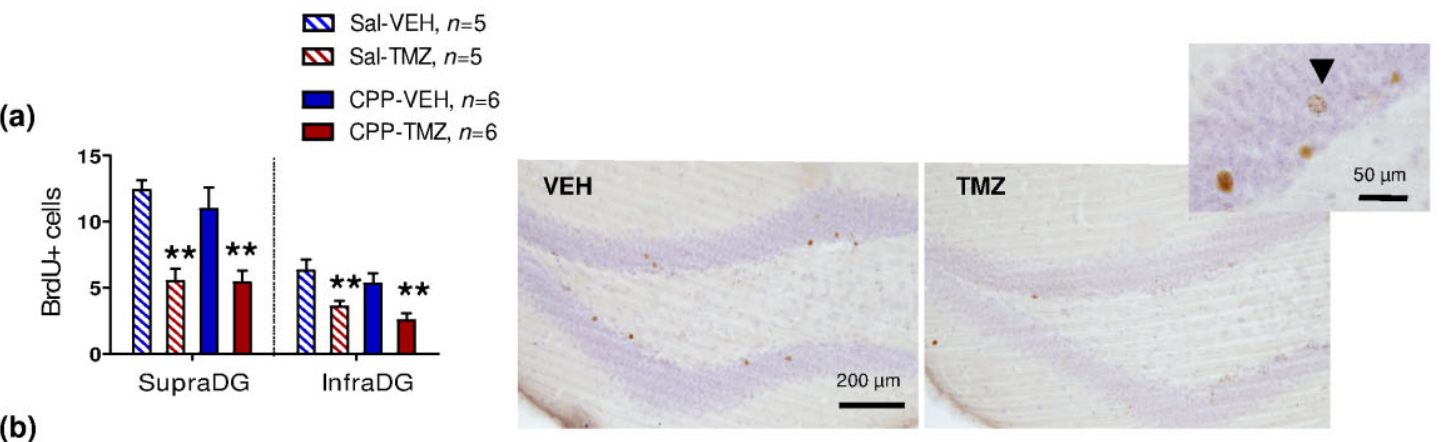

(b)
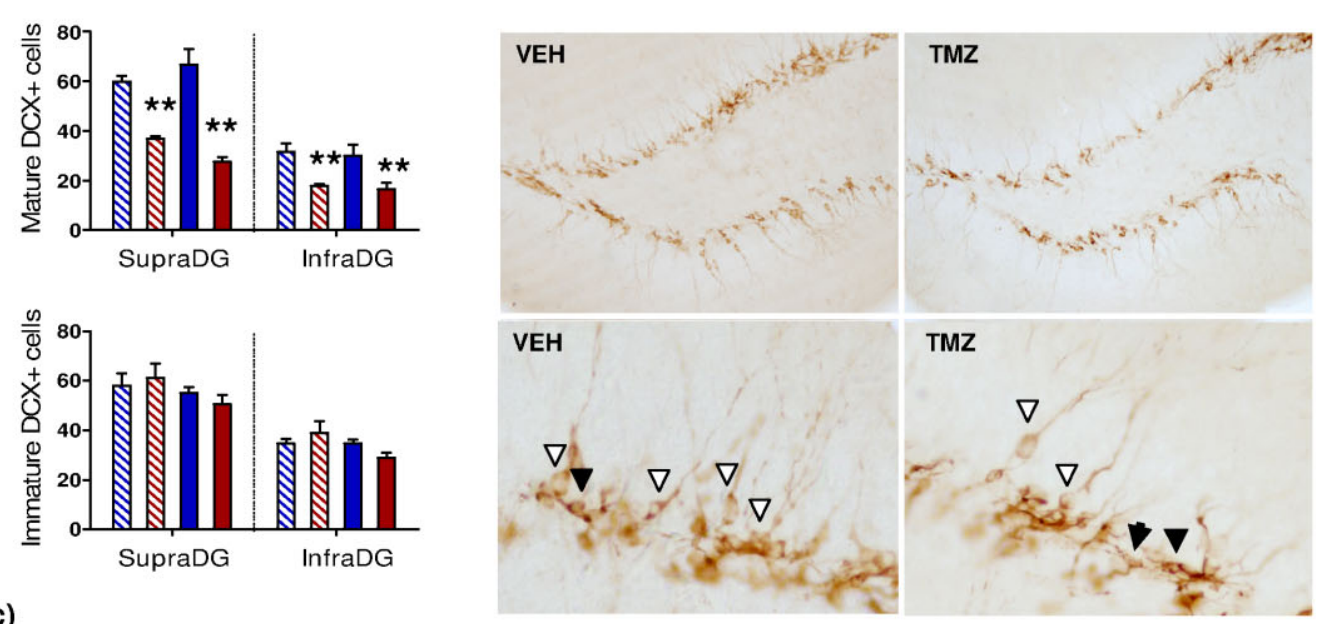

(c)
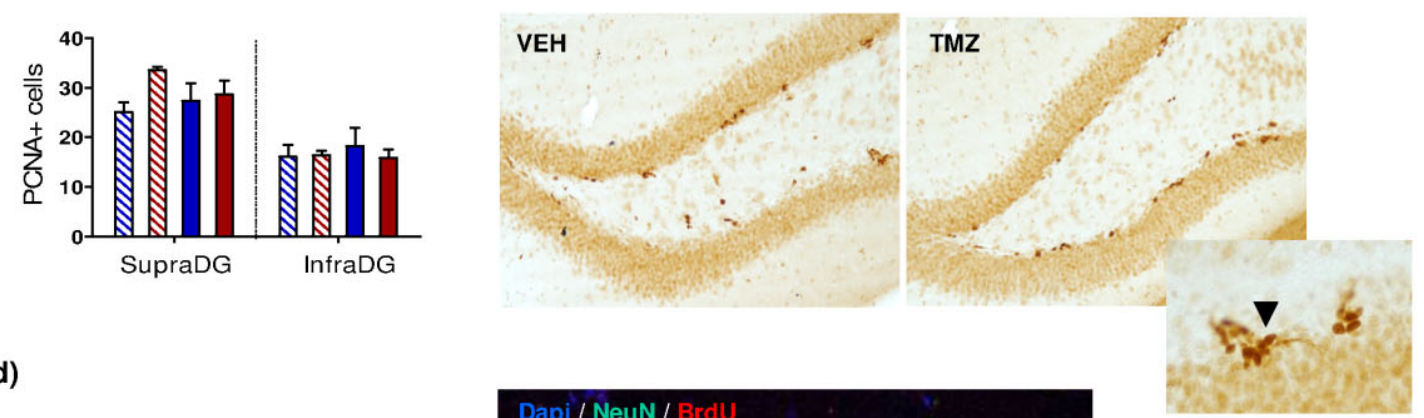

(d)
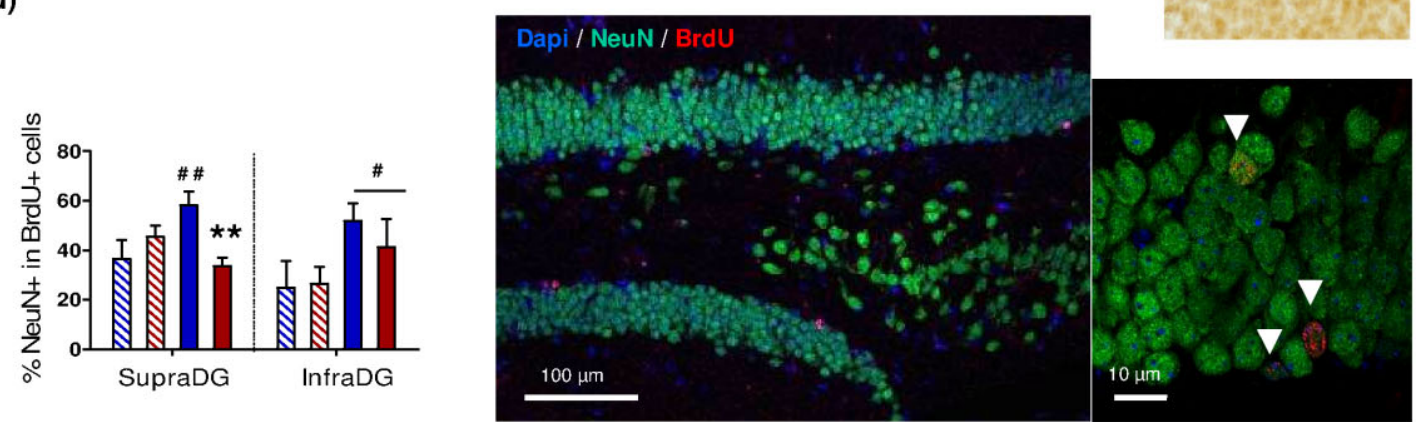

Figure 2 Reduction of adult hippocampal neurogenesis (mean number of positive cells per section \pm SEM) by TMZ. In both DG blades, TMZ-treated animals showed an almost 50 percent reduction in both the BrdU+ cells (a) and the DCX+ cells with mature morphology [i.e. with at least one prominent apical dendrite penetrating the granule cell layer; examples are indicated by white arrows in (b)]. Nevertheless, they had a normal number of DCX+ cells with immature morphology [i.e. with short or non-existent dendritic processes; examples are indicated by black arrows in (b)] and proliferating PCNA+ cells (c), showing a recovered neurogenic capacity at the time of perfusion. The images in $a, b$ and $c$ show representative staining of the DG in the VEH and TMZ treatment groups, and the arrows point to examples of positive labelling. The images do not distinguish the Sal from the CPP mice because no difference was found between these conditions. (d) Acquisition of the CPP increased the differentiation of the BrdU+ cells into mature neurons (mean \pm SEM), but this effect was potentiated in the CPP-VEH mice in the SupraDG. The image shows BrdU/NeuN co-labelling assessed with confocal microscopy. Difference versus the VEH group with the same treatment: $* * P \leq 0.00$ I; difference versus the Sal group: $\# P \leq 0.00$ I, \#\#P $\leq 0.00$ I. The scales in a are valid for $b$ and $c$ 

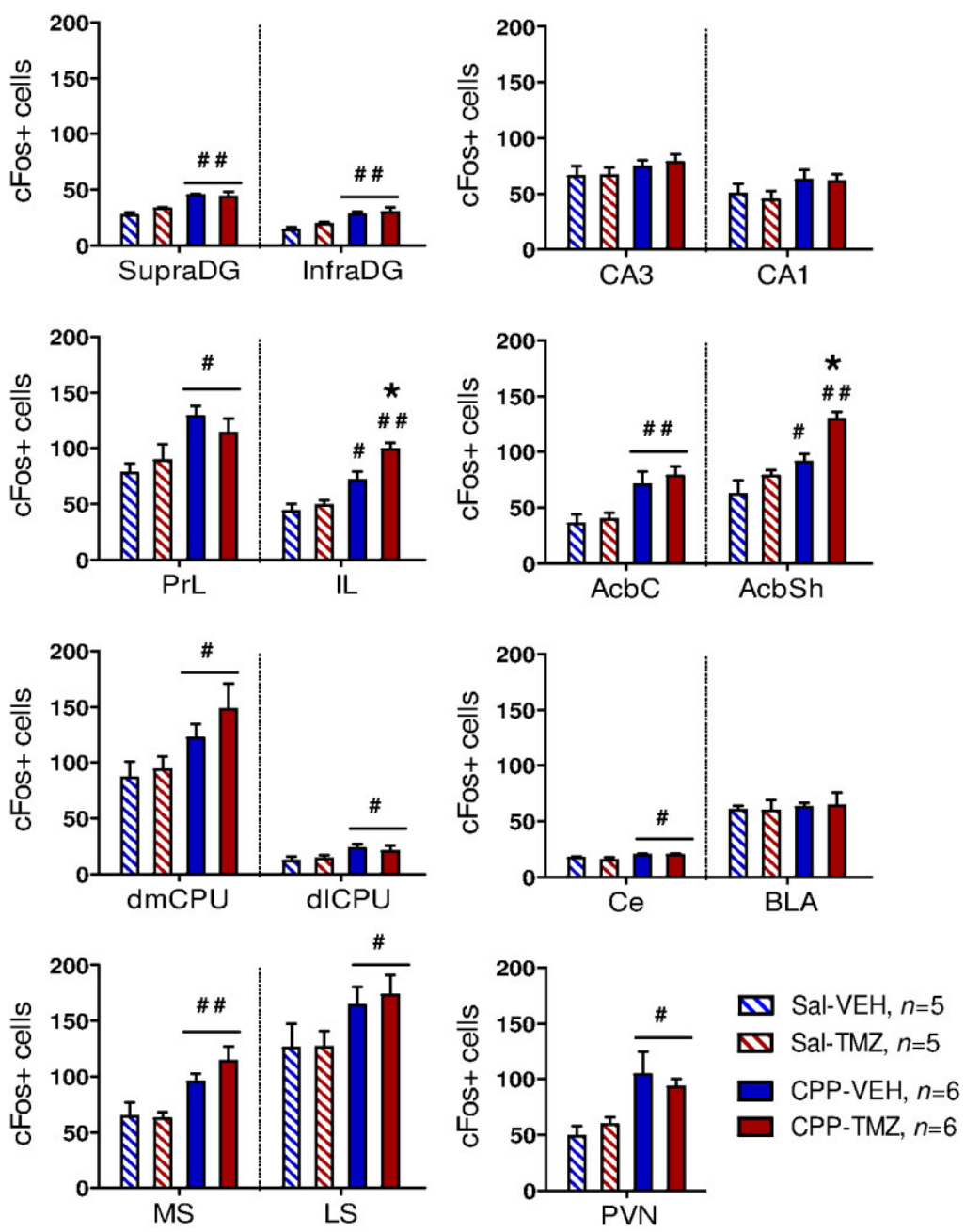

Figure 3 C-Fos expression (mean number of positive cells per section \pm SEM) after the CPP test in the CPP-VEH and CPP-TMZ mice compared with the saline condition. An effect of the cocaine treatment (Sal versus CPP) was found to be significant in all the assessed brain areas according to an ANOVA, with the exception of the $C A 3, C A I$ and $B L A$. In the $I L$ and AcbSh areas, c-Fos expression induced by cocaine seeking was greater for the CPPTMZ than for the CPP-VEH mice. Photos of representative staining are available in Supporting Information Fig. S4. Difference versus the $\mathrm{VEH}$ group with the same treatment: $* P \leq 0.05$; difference versus the Sal group: $\# P \leq 0.05, \# \# P \leq 0.00$ I

\section{TMZ treatment did not affect CPP expression}

The TMZ treatment had no effect on the acquisition of the cocaine-CPP, as both the cocaine-VEH and the cocaineTMZ mice displayed a similar and significant CPP-score in the test session, while the Sal-mice did not prefer any compartment ['cocaine': $F(1,54)=6.675$, $P=0.013$, 'session': $F(1,54)=16.305, \quad P=0.000$; 'cocaine $\times$ session': $F(1,54)=7.627, P=0.008$; comparisons are shown in Fig. 1b]. The analysis of the CPPscore every 4 minutes (Fig. 1c) also yielded no effect. Locomotion in the test session was similar for both treatments (data not shown).

Adult hippocampal neurogenesis was reduced by the TMZ treatment

A notable reduction of both BrdU+ cells and young DCX+ neurons with mature morphology was caused by the TMZ treatment in both DG blades ['treatment' effect for BrdU: SupraDG: $F(1,18)=30.257, P=0.000$; InfraDG: $F(1,18)=18.238, P=0.000$; Fig. 2a; 'treatment' effect for mature DCX: SupraDG: $F(1,18)=65.858, P=0.000$;
InfraDG: $F(1,18)=15.977, P=0.001$; Fig. 2b]. Nevertheless, the young DCX+ neurons with immature morphology (Fig. 2b) and the proliferating cells expressing PCNA (Fig. 2c) were not altered by TMZ.

No difference was found between the Sal and the CPP conditions in the BrdU, DCX or PCNA measures, indicating that cocaine did not deplete neurogenesis. On the contrary, groups that acquired the CPP showed increased differentiation of the BrdU+ cells into mature NeuN+ neurons (Fig. 2d). Interestingly, in the SupraDG, such enhancement was only noticeable for the CPP-VEH mice [SupraDG: 'treatment $\times$ CPP': $F(1,18)=11.516$, $P=0.003$; InfraDG: 'CPP': $\mathrm{F}(1,18)=4.882, P=0.040$; LSD is shown in Fig. 2d].

\section{C-Fos expression was increased in the CPP-TMZ mice}

Compared with the saline-treated mice, mice that expressed the cocaine-induced CPP showed increased c-Fos activity in most of the analysed brain areas, with the exception of CA1, CA3 and BLA ['CPP': SupraDG: $\quad F(1,18)=20.344, \quad P=0.000 ; \quad$ InfraDG: 
(a)

Functional brain circuit - VEH group

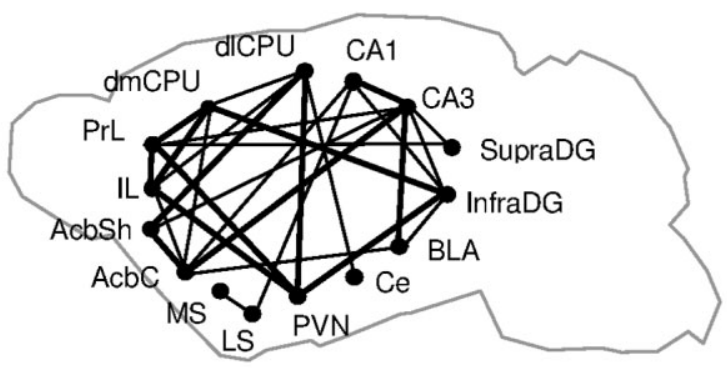

(b)

\begin{tabular}{|c|c|c|c|c|c|}
\hline & \multicolumn{2}{|c|}{ VEH } & \multicolumn{3}{|c|}{ TMZ } \\
\hline & $\begin{array}{c}\text { Factor } \\
1 \\
\end{array}$ & $\begin{array}{c}\text { Factor } \\
2 \\
\end{array}$ & $\begin{array}{c}\text { Factor } \\
1 \\
\end{array}$ & $\begin{array}{c}\text { Factor } \\
2 \\
\end{array}$ & $\begin{array}{c}\text { Factor } \\
3 \\
\end{array}$ \\
\hline SupraDG & .030 & .872 & .260 & -.134 & .908 \\
\hline InfraDG & .346 & .781 & -.103 & .236 & .933 \\
\hline CA & .189 & .817 & .813 & .222 & .469 \\
\hline PrL & .535 & .664 & .540 & .536 & -.345 \\
\hline IL & .860 & .371 & .008 & .976 & .132 \\
\hline Acb & .718 & .298 & .678 & .523 & .372 \\
\hline $\mathrm{dmCPU}$ & .741 & .576 & .772 & .565 & -.117 \\
\hline dlCPU & .947 & -.121 & .976 & .013 & .010 \\
\hline PVN & .657 & .547 & .393 & .769 & .061 \\
\hline Eigenvalue & 3.576 & 3.350 & 3.193 & 2.548 & 2.208 \\
\hline$\%$ Variance & 39.74 & 37.22 & 35.48 & 28.31 & 24.53 \\
\hline $\begin{array}{r}r \text { (factor } \\
\text { score-CPP } \\
\text { score) })\end{array}$ & $\begin{array}{c}.019 \\
P=.956\end{array}$ & $\begin{array}{c}.780^{*} \\
P=.005\end{array}$ & $\begin{array}{c}.088 \\
P=.797\end{array}$ & $\begin{array}{c}.695^{\star} \\
P=.018\end{array}$ & $\begin{array}{c}-.070 \\
P=.839\end{array}$ \\
\hline
\end{tabular}

Functional brain circuit - TMZ group

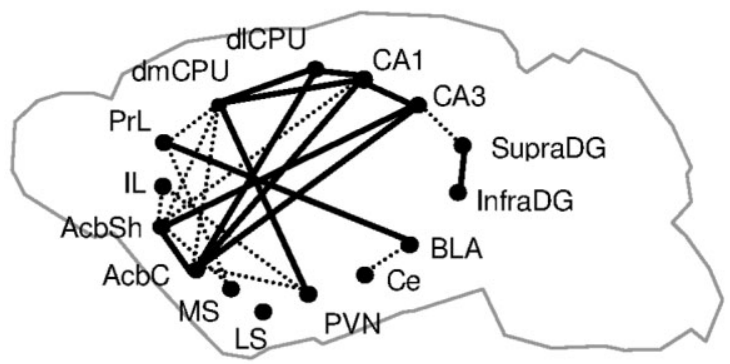

(c)
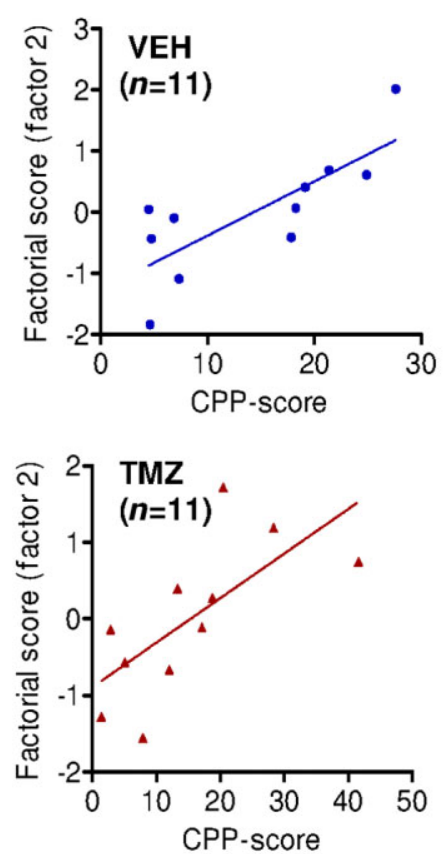

Figure 4 Functional brain circuits supporting CPP expression in VEH and TMZ mice. (a) Graphical representation of the functional brain circuits activated after the CPP test in VEH and TMZ mice. The inhibition of adult hippocampal neurogenesis decreased functional connectivity among the structures, especially in the DG. Significant Pearson's correlations between areas are represented as a connecting line $(P \leq 0.05$; $P \leq 0.0$ I are indicated by bold lines). All correlations were positive except the LS-CAI correlation in the VEH group, which was negative. Dotted correlations in the TMZ mice did not achieve the $q \leq 0.05$ criteria and thus have more than a 5 percent probability of being false positives (Supporting Information Methods SI; Supporting Information Table S2). (b) Factors resulting from the PCA in each experimental condition. Each factor represents a functional brain module that could potentially support behaviour. Some brain areas were removed from the analysis (Ce, BLA, MS, LS), and others were averaged into a single measure (CA, Acb) to achieve a sample suitable to perform the PCA (Supporting Information Methods SI). The areas included in a factor (with a factor loading $\geq 0.5$ in absolute value) are highlighted in bold, and the areas best representing a factor were those that loaded high on the factor and low on the others. Both the eigenvalue and the $\%$ variance (percent of the total variance explained by the factor) indicate the importance of a factor in explaining the total variability of the sample. The last row in the table shows Pearson's correlations between the factorial scores and the CPP-scores. Significant correlations are represented in c. Note that factor 2, which supported CPP behaviour, involved strong hippocampal participation in the VEH mice, including the SupraDG, InfraDG and CA areas. However, in TMZ animals, CPP expression was supported mainly by the IL and stress-related areas, such as the PVN, while the DG loaded in a factor separate from the other structures

$F(1,18)=14.789, P=0.001 ;$ PrL: $F(1,18)=9.927$, $P=0.006 ; \quad \mathrm{IL}: F(1,18)=37.831, P=0.000 ;$ AcbC: $F(1, \quad 18)=15.151, \quad P=0.001 ; \quad$ AcbSh: $F(1,18)=$ 24.355, $\quad P=0.000 ; \quad$ dmCPU: $\quad F(1, \quad 18)=6.996$, $P=0.017$; dlCPU: $F(1,18)=4.470, P=0.049$; Ce: $F(1,18)=4.872, P=0.041$; MS: $F(1,18)=16.848$, $P=0.001 ; \quad$ LS: $F(1,18)=6.452, \quad P=0.021 ; \quad P V N$ :
$F(1,18)=11.226, P=0.004$; Fig. 3; representative photographs are available in Supporting Information Fig. S4]. Importantly, the IL and the AcbSh also revealed an effect of the TMZ treatment, as the CPP-TMZ mice showed more c-Fos expression than the other groups ['treatment': IL: $F(1,18)=6.560, P=0.020$; AcbSh: $F(1,18)=11.164, P=0.004$; LSD is shown in Fig. 3]. 
The increased c-Fos activity in the CPP-TMZ mice could not be accounted for by the locomotion in the test session (which was similar to the locomotion in the CPPVEH mice, not shown) and was not found in a sample of cocaine-treated TMZ mice that showed a low CPP-score (Supporting Information Fig. S5), indicating that it was driven by drug seeking and not simply by cocaine administration.

The TMZ-treated mice engaged different neurobiological mechanisms for drug seeking

Correlation between neurogenesis-related measures and CPP expression. In the VEH-treated mice, CPP learning was associated with enhanced differentiation of the BrdU+ cells into mature NeuN+ neurons in the SupraDG, but this relationship was opposite for the TMZ animals (Supporting Information Table S1), suggesting distinct neurobiological mechanisms.

Functional brain circuits supporting CPP expression. The TMZ treatment modified the functional brain circuits activated after the CPP test. Pearson's correlations between brain areas (Supporting Information Table S2) revealed widespread functional connectivity in the VEH mice, with 29 significant correlations (Fig. 4a). However, the TMZ mice displayed reduced functional connectivity, yielding only 23 significant correlations, and 11 of them did not meet the $q$ criteria, which means they had more than a 5 percent probability of being false positives (Storey 2002) and should be interpreted with caution (Fig. 4a, dotted lines). Interestingly, reduced functional connectivity in the TMZ mice was most evident in the DG areas.

The PCA revealed a solution with two independent factors (i.e. functional brain modules) in the VEH mice that accounted for 76.96 percent of the total variance. Importantly, the factor supporting a correlation with the CPP-score (factor 2, Fig. 4b \& c) reflected hippocampal activity, as it involved a strong participation of the DG and CA areas (with a partial contribution of the PrL, dmCPU and PVN). For the TMZ mice, the PCA revealed three independent factors accounting for 88.32 percent of the total variance, where the DG formed a factor separate from the rest. The factor that showed a correlation with the CPP-score in the TMZ mice (factor 2) had no hippocampal participation but was strongly represented by the IL area (Fig. $4 \mathrm{~b} \& \mathrm{c}$ ), and the involvement of the Acb and PVN areas was also increased compared with the VEH-mice. Intriguingly, factor 2 in the TMZ mice, but not in the VEH mice, also correlated with locomotor activity in the test session $(r=0.720$, $P=0.012$ ).

\section{EXPERIMENT 2: ROLE OF ADULT HIPPOCAMPAL NEUROGENESIS IN CPP EXTINCTION AND COCAINE-INDUCED REINSTATEMENT}

\section{Methods}

The requirement of adult hippocampal neurogenesis for CPP extinction and cocaine-induced reinstatement was tested by means of two different experimental protocols: with neurogenesis inhibition occurring either previous to (PreTr groups) or after (PostTr groups) the CPP training (Fig. 5a and Supporting Information Methods S2).

The PreTr mice (PreTr-VEH, $n=9$; PreTr-TMZ, $n=9$ ) underwent the VEH or TMZ treatment and the CPP training exactly as described for mice in experiment 1 (Figs 1a \& 5a) and started extinction training the day after the test session. Mice in the PostTr groups (PostTr-VEH, $n=8$; PostTr-TMZ, $n=6$ ) underwent the CPP training at the same age and in the same conditions as the PreTr mice and then were divided into two similar groups to be administered the VEH or TMZ treatment (Fig. 5a) and were submitted to 21 days of abstinence before extinction training started.

In this way, both the PreTr and the PostTr mice underwent extinction training on days 13-28 and the cocaine-induced reinstatement session on day 29 after the last VEH/TMZ dose (Fig. 5a). Therefore, the age of the inhibited hippocampal neurons was the same for both conditions.

\section{Results}

Result showed that the behavioural protocol used (PreTr or PostTr) influenced extinction and the effect of the TMZ on this process. This was revealed by a repeatedmeasures ANOVA across the test and the 12 extinction sessions ['protocol': $F(1,28)=4.585, P=0,041$; 'day': $F(12,336)=11.499, P=0.000$; 'protocol $\times$ day $\times$ TMZ' tended towards significance: $F(12,336)=1.760, P=$ $0.054]$ and on extinction data normalized as the percent of change from the initial CPP, averaged in blocks of four sessions ['block': $F(2,56)=4.332, P=0.018$; 'protocol $\times$ TMZ': $\quad F(1,28)=4.793, P=0.037]$. Therefore, extinction was analysed separately for the PreTr and the PostTr mice. For mice in the PreTr protocol, TMZ delayed the extinction of the CPP behaviour ['day': $F(12,192)=$ 7.657, $P=0.000$; 'day $\times$ TMZ': $F(12,192)=1.892$, $P=0.037$; Fig. 5b], and a comparison of both treatments resulted in a significant difference for sessions $5-8[t(16)$ : -2.600; $P=0.019$ ], where only the PreTr-VEH mice had extinguished the CPP (Fig. 5d). On the other hand, the PostTr protocol seemed more demanding for extinction as the PostTr-VEH did not extinguish until sessions 9-12, but both treatments performed at once (Fig. $5 \mathrm{c} \& \mathrm{e}$ ). Nevertheless, thePostTr-TMZ animals showed increased CPP 
(a)

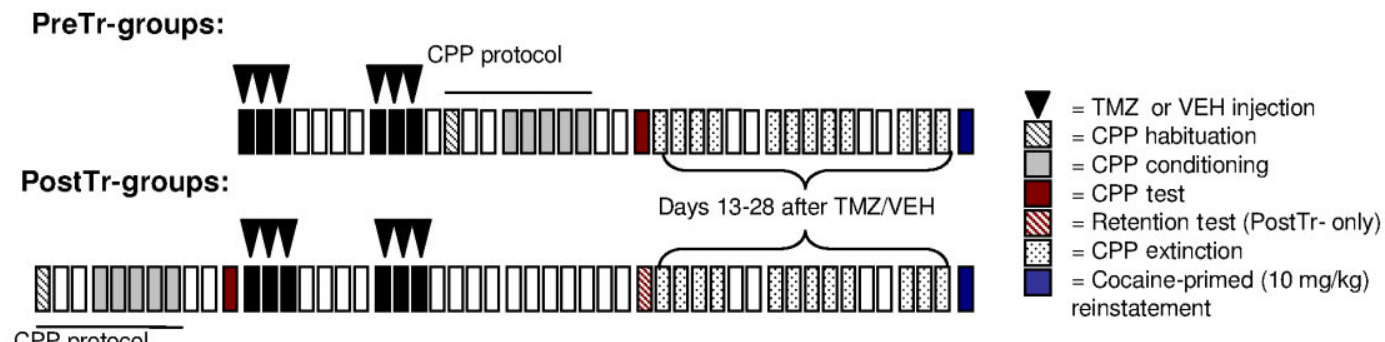

(b)

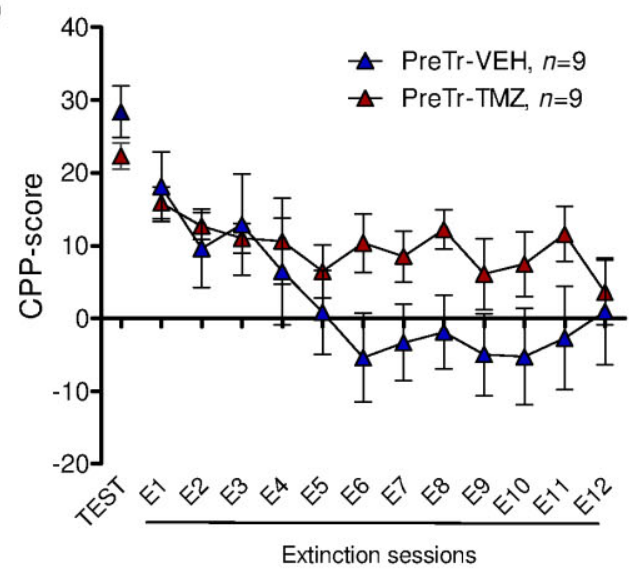

(d)

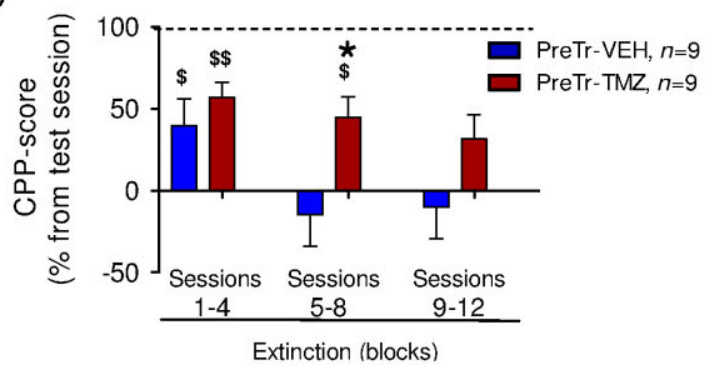

(f)

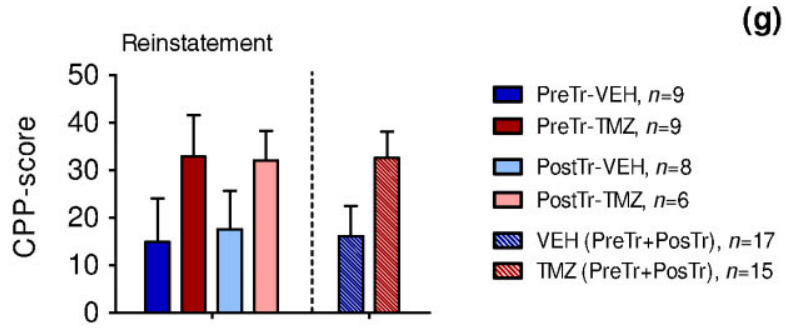

(c)

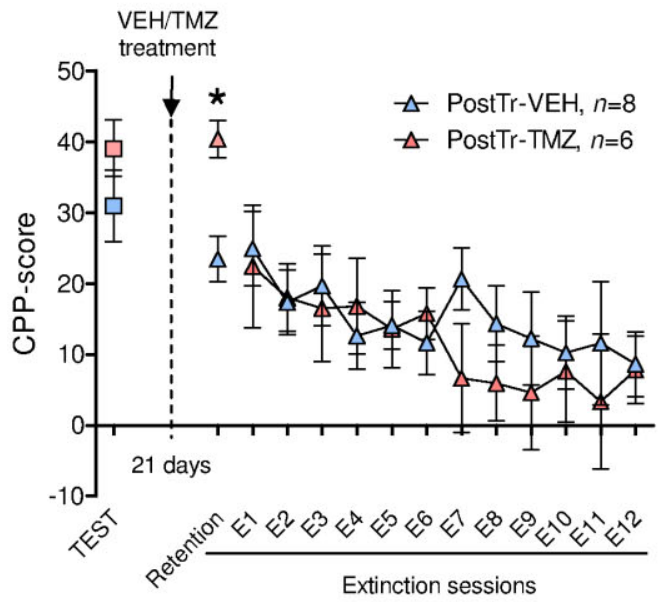

(e)

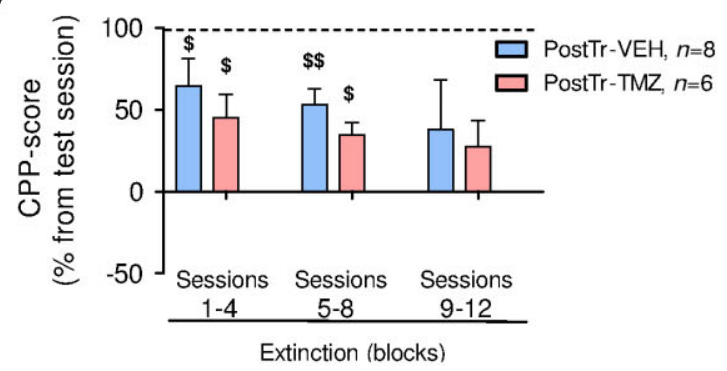

(g)

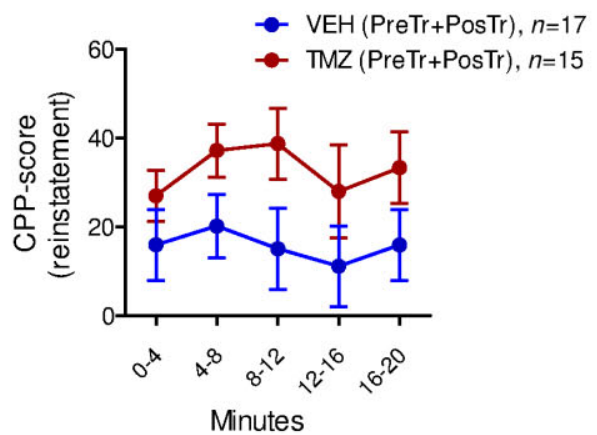

Figure 5 Effect of adult hippocampal neurogenesis inhibition on the extinction and cocaine-induced reinstatement of the conditioned place preference. (a) Experimental protocol used for Experiment 2. The TMZ treatment took place before (PreTr groups, which used a similar protocol as animals in experiment I) or after (PostTr groups) the CPP conditioning. Importantly, the age of the cells affected by the TMZ treatment was similar in both the PreTr and the PostTr protocols when extinction started. (b-e) Extinction data shown as the CPP-score per session ( $b$ and c) and as a percent change from the initial acquisition (d) and (e). Inhibition of adult hippocampal neurogenesis delayed extinction of the CPP in the PreTr condition (b) and (d) but not in the PostTr mice (c and e). However, PostTr-TMZ animals had enhanced long-term CPP retention (c). ( $\mathrm{f}$ and g) Cocaine-induced reinstatement was not affected by the behavioural protocol, but it increased after TMZ treatment. Difference versus the VEH group with the same treatment: $* P<0.05$; difference from zero: $\$ P<0.05, \$ \$ P<0.001$. Data are expressed as the mean \pm SEM 
when tested in the long-term retention session [t(12): $-4.103, P=0.001]$.

Cocaine-induced reinstatement was not influenced by the behavioural protocol employed, so data from the PreTr and the PostTr mice were merged for analysis (Fig. 5f). The results suggested that the TMZ treatment increased cocaine-induced reinstatement. This effect was nearly significant when the CPP-score was considered as a unique measure for the whole session [t(30): -1.972 ; $P=0.058$, Fig. 5f] and was significant when the session was analysed in intervals of every 4 minutes ['TMZ': $F(1,30)=4.394, P=0.045$, Fig. 5g]. Analysis of the extinction and reinstatement data expressed as seconds in the cocaine-paired compartment confirmed the results reported by the CPP-score (Supporting Information Fig. S6).

\section{DISCUSSION}

This study investigated the involvement of adult hippocampal neurogenesis in the cocaine-induced CPP model. A main result is that reducing the number of new hippocampal neurons before CPP acquisition altered the addiction-related functional brain circuits triggered to express cocaine seeking in the drug-paired context. In addition, neurogenesis reduction increased cocaineseeking behaviour. While animals with reduced neurogenesis normally acquired the drug-contextual associations, they were harder to extinguish. However, if neurogenesis was inhibited after CPP acquisition, neurogenesis-reduced animals showed increased longterm CPP retention, but no effect on extinction was found, suggesting that some of the roles of the newly born neurons depend on the timing of their generation. Finally, reduction of adult hippocampal neurogenesis increased cocaine-induced CPP reinstatement in both behavioural protocols used. These results support that reduced adult hippocampal neurogenesis yields significant neurobiological changes that may be relevant to addiction and increases engagement in drug-seeking behaviours.

The first experiment confirmed that the TMZ treatment was effective at inhibiting adult hippocampal neurogenesis in the absence of detectable inflammation or behavioural alterations in exploratory or anxiety-like measures. Because the TMZ-treated mice had almost half of the BrdU+ cells and DCX+ neurons with mature morphology but showed a normal number of proliferating cells $(\mathrm{PCNA}+$ cells and immature DCX+ neurons that are usually still able to undergo proliferation; Plumpe et al. 2006), it is suggested that the TMZ caused a low-harmful, transient effect so the DG had recovered its neurogenic capacity at the time of the histological analysis (i.e. 12 days after the last TMZ dose). On the other hand, adult hippocampal neurogenesis was not affected by cocaine administration. Despite the abundant evidence regarding the neurogenesis-inhibitory effects of cocaine in rats (reviewed in Jun et al. 2012), this topic requires further investigation in mice. The only available experiment (Lloyd et al. 2010) contrarily reports that cocaine transitorily increased cell proliferation in the mouse DG and used a chronic cocaine treatment $(20 \mathrm{mg} / \mathrm{kg}$ per day for 28 days) not comparable to this study.

Despite the adult hippocampal neurogenesis reduction, the TMZ-treated mice showed drug-seeking behaviour, but the analysis of the functional brain circuits confirmed that different neural pathways were activated in the neurogenesis-reduced condition. In this regard, functional connectivity among the addiction-related areas was abundant in the VEH mice, and CPP expression relied heavily on hippocampal activation. This outcome is not surprising because the hippocampus, specifically the DG, is essential for the acquisition and expression of cocaine-induced CPP (Meyers et al. 2006; Hernandez-Rabaza et al. 2008) as well as for other forms of spatial learning and stimuli-context associations (Bannerman et al. 2004). However, when hippocampal functioning is compromised by neurogenesis depletion, learning and addictive processes may reorganize the underlying functional circuitry and enhance the recruitment of extrahippocampal areas (Chambers 2013). In agreement with this, the TMZ treatment greatly impaired the functional connectivity of the DG and prevented the hippocampus from supporting drug seeking. Instead, drug seeking strongly depended on the IL area, and the involvement of the Acb and PVN was also increased. The disruption of hippocampal connectivity could possibly enhance IL participation because following hippocampal damage, the mPFC undergoes a fast - though incomplete-compensation of the hippocampal function for spatial learning (Lee \& Kesner 2003). The mPFC also has a crucial role in drug addiction, as it modulates the extinction of drug-related memories, drug relapse, impulsivity and the turning of addictive behaviours into habits (Peters, Pattij \& De Vries 2013). Importantly, the mPFC projects to the accumbens to support both drug seeking and craving, but there is a functional dissociation, so the PrL potentiates addiction-related responses and they are reduced by the IL area (Peters et al. 2013; Ma et al. 2014). Specifically, the IL preferentially projects to the AcbSh to suppress cocaine seeking and craving (Peters et al. 2009, 2013; Ma et al. 2014), and this IL-AcbSh circuit seems dysregulated in the CPP-TMZ mice, given their abnormally increased c-Fos activity in both areas. The IL is also a key node where the addiction circuits overlap with those for fear and stress, explaining the enhanced involvement of the PVN to support drug seeking in the TMZ mice (Peters et al. 2009; Koob \& Zorrilla 2010). The 
core elements of the brain's stress systems also take part in drug addiction, such as in stress-induced relapse or in the negative emotions associated with drug withdrawal (Koob \& Zorrilla 2010).

The aforementioned results show that adult-born hippocampal neurons sculpt the addiction-related circuits, suggesting that they are incorporated in these networks at some point. The new neurons suppressed in this experiment were a young population 2-3 weeks old on the day of the CPP Test. However, functional integration into the existing brain circuitry may have taken place at an early maturational stage. At the first week of age, new neurons already project long axons to the CA3 area and dendrites to the DG's molecular layer, so from the second week, they exhibit dendritic spines and functional integration, receiving direct presynaptic input from extrahippocampal areas (Deng, Aimone \& Gage 2010; Castilla-Ortega et al. 2011b; Deshpande et al. 2013). In addition, hippocampal neurons younger than 3 weeks are more likely to be modulated by hippocampal-relevant experiences because of their enhanced plasticity and unique electrophysiological properties (Tashiro, Makino \& Gage 2007; Castilla-Ortega et al. 2011b), and they also express early immediate genes after hippocampaldependent tasks (Agis-Balboa et al. 2011; Vukovic et al. 2013). After specific removal of the population of young neurons, hippocampal-dependent learning is impaired (Deng et al. 2009; Goodman et al. 2010; Vukovic et al. 2013) and new memories take longer to become independent of the hippocampus (Kitamura et al. 2009), supporting a contribution of hippocampal-cortical connectivity.

Although the alternate functional brain circuit engaged in neurogenesis-reduced conditions sufficed to support CPP expression, the subsequent extinction of drug-seeking behaviour was delayed as revealed in the second experiment with the PreTr-TMZ mice. This agrees with studies where fear-contextual associations are acquired in the absence of adult neurogenesis but are harder to extinguish (Deng et al. 2009; Pan et al. 2012). However, extinction was preserved when the neurons generated after CPP learning (PostTr mice) were inhibited. The PostTr protocol would have been expected to stress the role of the adult-born neurons in extinction because they are especially involved in highly demanding tasks (Castilla-Ortega et al. 2011b) and extinction seemed to be more difficult in the PostTr condition (probably because it included a prolonged abstinence that increased drug-craving incubation; e.g. Karlsson et al. 2013; a similar outcome is shown in Mustroph et al. (2011) for control mice). This may suggest that the extinction deficit was specifically caused by preventing new neurons' engagement in CPP acquisition. Nevertheless, hippocampal neurons generated after acquiring the drug-context associations would also support a relevant role because the PostTr-TMZ mice potentiated long-term CPP retention, and, more importantly, cocaine-induced reinstatement was enhanced in the TMZ mice independently of the behavioural protocol used (PreTr or PostTr).

These results demonstrate a role of adult hippocampal neurogenesis in drug-seeking behaviour in the CPP model. Nevertheless, it must be noted that the CPP paradigm entails both cognitive and motivational components, as drug seeking is not only driven by remembering the drug-contextual association but also by its perceived reinforcing value. On the one hand, adult hippocampal neurogenesis has a widely reported role in hippocampaldependent memory, as revealed in multiple behavioural tasks (Deng et al. 2010; Leuner \& Gould 2010; Castilla-Ortega et al. 2011b). In contextual fearconditioning paradigms, ablation of adult hippocampal neurogenesis may impair both the acquisition and extinction of the fear-contextual associations (Castilla-Ortega et al. 2011b) and, interestingly, inhibition of the neurons proliferating after fear conditioning potentiates its longterm retention, pointing to their requirement for memory forgetting and clearance (Akers et al. 2014). Therefore, results reported here for the extinction and retention of drug-contextual associations may not be exclusive to drug-related memories and could be explained, at least in part, by the role of adult hippocampal neurogenesis in contextual learning. On the other hand, adult hippocampal neurogenesis is involved in non-cognitive processes such as emotional control by inhibiting the hypothalamic-pituitary-adrenal axis (Snyder et al. 2011). Considering that adult neurogenesis reduction increases cocaine reward because neurogenesis-reduced rats self-administer more cocaine (Noonan et al. 2010), an enhanced craving for cocaine could be present in the TMZ mice and also contribute to their behaviour.

Therefore, the role of adult hippocampal neurogenesis in cocaine-induced CPP behaviour is complex as it likely entails both cognitive and motivational elements and depends on numerous factors such as the behaviour studied (acquisition, extinction or cocaine-induced reinstatement) and the timing of neurogenesis inhibition (before or after CPP learning). This may explain why previous reports have failed to find significant effects in cocaine-induced CPP after neurogenesis inhibition. In the study by Brown et al. (2010b), rats with a profound adult neurogenesis depletion acquired the cocaineinduced CPP, as we found in our mice, but CPP extinction and reinstatement were not evaluated. In the case of Mustroph et al. (2015), CPP extinction was tested, but neurogenesis inhibition occurred after the CPP was acquired, and the new neurons generated after CPP conditioning do not appear to be involved in its extinction, according to our results. An apparent contradiction with 
our study is that mice in Mustroph et al. (2015) did not show enhanced long-term CPP retention, so methodological differences such as in the conditioning training used or in the age of the new neurons inhibited could possibly explain this outcome (Castilla-Ortega et al. 2011b). Other experiments have suppressed adult hippocampal neurogenesis in the cocaine selfadministration paradigm (Noonan et al. 2010; Deschaux et al. 2014). The first (Noonan et al. 2010) showed that rats irradiated after self-administration were impaired in contextual extinction and tended to increase cocaineand stress-induced reinstatement, while the second (Deschaux et al. 2014) showed increased cocaineinduced reinstatement after applying low-frequency stimulation during extinction training. While these studies are hardly comparable to our CPP report (as both paradigms may differ greatly; Bardo \& Bevins 2000), they agree in that reduced adult hippocampal neurogenesis would enhance drug-seeking behaviour.

Overall, evidence from animal studies support reduced adult hippocampal neurogenesis as a potential vulnerability factor for drug addiction. Focusing on the altered functional brain circuits triggered for addiction in the absence of adult neurogenesis may help to identify brain areas relevant for therapeutic purposes. This is especially important in the prefrontal cortex-ventral/dorsal striatum circuitry where habit-forming drugs might impose non-declarative implicit memories that sustain drug-seeking behaviour.

\section{Acknowledgements}

The authors gratefully acknowledge IBIMA joint services, common support structures for research (ECAI) of General Services, Microscopy and Animal Experimentation, for management, immunohistology and maintenance of mice, respectively. Likewise, we are obliged to central microscopy facilities at Universidad de Málaga for confocal and electron microscopy.

We are grateful to Ana Isabel Gómez Conde and Juan Gómez Repiso for their technical contribution, and to David Navas Fernández for his assistance in confocal microscopy. The authors also acknowledge the staff of the animal facility at the University of Málaga for their kind assistance during the performance of the behavioral experiments.

This research was funded by Spanish Ministry of Economy and Competitiveness (PSI2013-44901-P, co-funded by European Research Development Fund -ERDF-, to L.J.S; Subprograma RETICS Red de Trastornos Adictivos RD12/0028/0001, to F.R.F; P10/02514 02514 -co-funded by ERDF-, to G.E-T); Andalusian Ministries of Health and Economy, Innovation, Science and Employment (SEJ-4515, to L.J.S; CTS433, PI-0823-
2012, PI-0228-2013 and PI45403 to F.R.F; CTS643 and Nicolás Monardes Programme, to G.E-T) and Spanish Ministry of Health, Social Services and Equality and Plan Nacional sobre Drogas (049/2009 and 049/2013 to F.R.F). Author E.C.-O. holds a 'Sara Borrell' research contract from the Spanish Carlos III Health Institute, Spanish Ministry of Economy and Competitiveness (grant number CD12/00455). Author D.L.G-M. holds a 'FPU' grant from the Spanish Ministry of Education, Culture and Sports (grant number FPU13/04819). The confocal microscope was acquired by the University of Málaga with ERDF funds.

\section{Authors Contribution}

$\mathrm{ECO}, \mathrm{EB}, \mathrm{FRF}$ and LJS were responsible of the concept and design of the study. ECO, EB, AS, DLGM, MP, GET and FJP contributed to data collection and/or analysis. ECO and LJS wrote the manuscript, which was critically reviewed by FRF for important intellectual content. All authors critically reviewed content and approved the final version for publication.

\section{References}

Agis-Balboa RC, Arcos-Diaz D, Wittnam J, Govindarajan N, Blom K, Burkhardt S, Haladyniak U, Agbemenyah HY, Zovoilis A, Salinas-Riester G, Opitz L, Sananbenesi F, Fischer A (2011) A hippocampal insulin-growth factor 2 pathway regulates the extinction of fear memories. EMBO J 30:4071-4083.

Akers KG, Martinez-Canabal A, Restivo L, Yiu AP, De Cristofaro A, Hsiang HL, Wheeler AL, Guskjolen A, Niibori Y, Shoji H, Ohira K, Richards BA, Miyakawa T, Josselyn SA, Frankland PW (2014) Hippocampal neurogenesis regulates forgetting during adulthood and infancy. Science 344:598-602.

Balaban CD, Ogburn SW, Warshafsky SG, Ahmed A, Yates BJ (2014) Identification of neural networks that contribute to motion sickness through principal components analysis of fos labeling induced by galvanic vestibular stimulation. PLOS ONE 9:e86730.

Bannerman DM, Rawlins JN, McHugh SB, Deacon RM, Yee BK, Bast T, Zhang WN, Pothuizen HH, Feldon J (2004) Regional dissociations within the hippocampus - memory and anxiety. Neurosci Biobehav Rev 28:273-283.

Bardo MT, Bevins RA (2000) Conditioned place preference: what does it add to our preclinical understanding of drug reward? Psychopharmacology (Berl) 153:31-43.

Bilbao A, Blanco E, Luque-Rojas MJ, Suarez J, Palomino A, Vida M, Araos P, Bermudez-Silva FJ, Fernandez-Espejo E, Spanagel R, Rodriguez de Fonseca F (2013) Oleoylethanolamide dosedependently attenuates cocaine-induced behaviours through a PPARalpha receptor-independent mechanism. Addict Biol 18:78-87.

Blanco-Calvo E, Rivera P, Arrabal S, Vargas A, Pavon FJ, Serrano A, Castilla-Ortega E, Galeano P, Rubio L, Suarez J, Rodriguez de Fonseca F (2014) Pharmacological blockade of either cannabinoid $\mathrm{CB} 1$ or $\mathrm{CB} 2$ receptors prevents both cocaine-induced conditioned locomotion and cocaineinduced reduction of cell proliferation in the hippocampus of adult male rat. Front Integr Neurosci 7:106. doi: 10.3389/ fnint.2013.00106. 
Brown JP, Couillard-Despres S, Cooper-Kuhn CM, Winkler J, Aigner L, Kuhn HG (2003) Transient expression of doublecortin during adult neurogenesis. J Comp Neurol 467:1-10.

Brown RM, Short JL, Lawrence AJ (2010a) Identification of brain nuclei implicated in cocaine-primed reinstatement of conditioned place preference: a behaviour dissociable from sensitization. PLoS ONE 5:e15889.

Brown TE, Lee BR, Ryu V, Herzog T, Czaja K, Dong Y (2010b) Reducing hippocampal cell proliferation in the adult rat does not prevent the acquisition of cocaine-induced conditioned place preference. Neurosci Lett 481:41-46.

Canales JJ (2007) Adult neurogenesis and the memories of drug addiction. Eur Arch Psychiatry Clin Neurosci 257:261-270.

Castilla-Ortega E, Hoyo-Becerra C, Pedraza C, Chun J, Rodriguez De Fonseca F, Estivill-Torrus G, Santin LJ (2011a) Aggravation of chronic stress effects on hippocampal neurogenesis and spatial memory in LPA(1) receptor knockout mice. PLoS ONE 6:e25522.

Castilla-Ortega E, Pedraza C, Chun J, de Fonseca FR, Estivill-Torrus G, Santin LJ (2012) Hippocampal c-Fos activation in normal and LPA(1)-null mice after two object recognition tasks with different memory demands. Behav Brain Res 232:400-405.

Castilla-Ortega E, Pedraza C, Estivill-Torrus G, Santin LJ (2011b) When is adult hippocampal neurogenesis necessary for learning? evidence from animal research. Rev Neurosci 22:267283.

Castilla-Ortega E, Rosell-Valle C, Blanco E, Pedraza C, Chun J, Rodriguez de Fonseca F, Estivill-Torrus G, Santin LJ (2013) Reduced wheel running and blunted effects of voluntary exercise in LPA1-null mice: the importance of assessing the amount of running in transgenic mice studies. Neurosci Res 77:170-179.

Castilla-Ortega E, Rosell-Valle C, Pedraza C, Rodriguez de Fonseca F, Estivill-Torrus G, Santin LJ (2014) Voluntary exercise followed by chronic stress strikingly increases mature adult-born hippocampal neurons and prevents stress-induced deficits in 'what-when-where' memory. Neurobiol Learn Mem 109:62-73.

Castilla-Ortega E, Sanchez-Lopez J, Hoyo-Becerra C, Matas-Rico E, Zambrana-Infantes E, Chun J, De Fonseca FR, Pedraza C, Estivill-Torrus G, Santin LJ (2010) Exploratory, anxiety and spatial memory impairments are dissociated in mice lacking the LPA1 receptor. Neurobiol Learn Mem 94:73-82.

Chambers RA (2013) Adult hippocampal neurogenesis in the pathogenesis of addiction and dual diagnosis disorders. Drug Alcohol Depend 130:1-12.

Cleva RM, Gass JT (2010) Neuroanatomical structures underlying the extinction of drug-seeking behavior. Open Addict J 3:63-75.

Deng W, Aimone JB, Gage FH (2010) New neurons and new memories: how does adult hippocampal neurogenesis affect learning and memory? Nat Rev Neurosci 11:339-350.

Deng W, Saxe MD, Gallina IS, Gage FH (2009) Adult-born hippocampal dentate granule cells undergoing maturation modulate learning and memory in the brain. J Neurosci 29:13532-13542.

Deschaux O, Vendruscolo LF, Schlosburg JE, Diaz-Aguilar L, Yuan CJ, Sobieraj JC, George O, Koob GF, Mandyam CD (2014) Hippocampal neurogenesis protects against cocaine-primed relapse. Addict Biol 19:562-574.

Deshpande A, Bergami M, Ghanem A, Conzelmann KK, Lepier A, Gotz M, Berninger B (2013) Retrograde monosynaptic tracing reveals the temporal evolution of inputs onto new neurons in the adult dentate gyrus and olfactory bulb. Proc Natl Acad Sci U S A 110:E1152-E1161.

Fuchs RA, Eaddy JL, Su ZI, Bell GH (2007) Interactions of the basolateral amygdala with the dorsal hippocampus and dorsomedial prefrontal cortex regulate drug context-induced reinstatement of cocaine-seeking in rats. Eur J Neurosci 26:487-498.

Garthe A, Behr J, Kempermann G (2009) Adult-generated hippocampal neurons allow the flexible use of spatially precise learning strategies. PLoS ONE 4:e5464.

Geil CR, Hayes DM, McClain JA, Liput DJ, Marshall SA, Chen KY, Nixon K (2014) Alcohol and adult hippocampal neurogenesis: promiscuous drug, wanton effects. Prog Neuropsychopharmacol Biol Psychiatry 54:103-113.

Goodman T, Trouche S, Massou I, Verret L, Zerwas M, Roullet P, Rampon C (2010) Young hippocampal neurons are critical for recent and remote spatial memory in adult mice. Neuroscience 171:769-778.

Hernandez-Rabaza V, Hontecillas-Prieto L, Velazquez-Sanchez C, Ferragud A, Perez-Villaba A, Arcusa A, Barcia JA, Trejo JL, Canales JJ (2008) The hippocampal dentate gyrus is essential for generating contextual memories of fear and drug-induced reward. Neurobiol Learn Mem 90:553-559.

Hyman SE (2005) Addiction: a disease of learning and memory. Am J Psychiatry 162:1414-1422.

Jinno S (2011) Topographic differences in adult neurogenesis in the mouse hippocampus: a stereology-based study using endogenous markers. Hippocampus 21:467-480.

Jun H, Mohammed Qasim Hussaini S, Rigby MJ, Jang MH (2012) Functional role of adult hippocampal neurogenesis as a therapeutic strategy for mental disorders. Neural Plast 2012:854285. doi: 10.1155/2012/854285.

Karila L, Petit A, Lowenstein W, Reynaud M (2012) Diagnosis and consequences of cocaine addiction. Curr Med Chem 19:5612-5618.

Karlsson RM, Kircher DM, Shaham Y, O'Donnell P (2013) Exaggerated cue-induced reinstatement of cocaine seeking but not incubation of cocaine craving in a developmental rat model of schizophrenia. Psychopharmacology (Berl) 226:45-51.

Kitamura T, Saitoh Y, Takashima N, Murayama A, Niibori Y, Ageta H, Sekiguchi M, Sugiyama H, Inokuchi K (2009) Adult neurogenesis modulates the hippocampus-dependent period of associative fear memory. Cell 139:814-827.

Koob GF, Volkow ND (2010) Neurocircuitry of addiction. Neuropsychopharmacology 35:217-238.

Koob GF, Zorrilla EP (2010) Neurobiological mechanisms of addiction: focus on corticotropin-releasing factor. Curr Opin Investig Drugs 11:63-71.

Lee I, Kesner RP (2003) Time-dependent relationship between the dorsal hippocampus and the prefrontal cortex in spatial memory. J Neurosci 23:1517-1523.

Leger M, Quiedeville A, Paizanis E, Natkunarajah S, Freret T, Boulouard M, Schumann-Bard P (2012) Environmental enrichment enhances episodic-like memory in association with a modified neuronal activation profile in adult mice. PLoS ONE 7:e48043.

Leuner B, Gould E (2010) Structural plasticity and hippocampal function. Annu Rev Psychol 61:111-140, C111-113.

Lloyd SA, Balest ZR, Corotto FS, Smeyne RJ (2010) Cocaine selectively increases proliferation in the adult murine hippocampus. Neurosci Lett 485:112-116.

Ma YY, Lee BR, Wang X, Guo C, Liu L, Cui R, Lan Y, Balcita-Pedicino JJ, Wolf ME, Sesack SR, Shaham Y, Schluter OM, Huang YH, Dong Y (2014) Bidirectional modulation of 
incubation of cocaine craving by silent synapse-based remodeling of prefrontal cortex to accumbens projections. Neuron 83:1453-1467.

Mandyam CD, Koob GF (2012) The addicted brain craves new neurons: putative role for adult-born progenitors in promoting recovery. Trends Neurosci 35:250-260.

Martinez-Canabal A, Akers KG, Josselyn SA, Frankland PW (2013) Age-dependent effects of hippocampal neurogenesis suppression on spatial learning. Hippocampus 23:66-74.

Meyers RA, Zavala AR, Speer CM, Neisewander JL (2006) Dorsal hippocampus inhibition disrupts acquisition and expression, but not consolidation, of cocaine conditioned place preference. Behav Neurosci 120:401-412.

Mustroph ML, Merritt JR, Holloway AL, Pinardo H, Miller DS, Kilby CN, Bucko P, Wyer A, Rhodes JS (2015) Increased adult hippocampal neurogenesis is not necessary for wheel running to abolish conditioned place preference for cocaine in mice. Eur J Neurosci 41:216-226.

Mustroph ML, Stobaugh DJ, Miller DS, DeYoung EK, Rhodes JS (2011) Wheel running can accelerate or delay extinction of conditioned place preference for cocaine in male C57BL/6J mice, depending on timing of wheel access. Eur J Neurosci 34:1161-1169.

Niibori Y, Yu TS, Epp JR, Akers KG, Josselyn SA, Frankland PW (2012) Suppression of adult neurogenesis impairs population coding of similar contexts in hippocampal CA3 region. Nat Commun 3:1253. doi: 10.1038/ncomms2261.

Nokia MS, Anderson ML, Shors TJ (2012) Chemotherapy disrupts learning, neurogenesis and theta activity in the adult brain. Eur J Neurosci 36:3521-3530.

Noonan MA, Bulin SE, Fuller DC, Eisch AJ (2010) Reduction of adult hippocampal neurogenesis confers vulnerability in an animal model of cocaine addiction. J Neurosci 30:304-315.

Pan YW, Chan GC, Kuo CT, Storm DR, Xia Z (2012) Inhibition of adult neurogenesis by inducible and targeted deletion of ERK 5 mitogen-activated protein kinase specifically in adult neurogenic regions impairs contextual fear extinction and remote fear memory. J Neurosci 32:6444-6455.

Peters J, Kalivas PW, Quirk GJ (2009) Extinction circuits for fear and addiction overlap in prefrontal cortex. Learn Mem 16:279-288.

Peters J, Pattij T, De Vries TJ (2013) Targeting cocaine versus heroin memories: divergent roles within ventromedial prefrontal cortex. Trends Pharmacol Sci 34:689-695.

Plumpe T, Ehninger D, Steiner B, Klempin F, Jessberger S, Brandt M, Romer B, Rodriguez GR, Kronenberg G, Kempermann G (2006) Variability of doublecortin-associated dendrite maturation in adult hippocampal neurogenesis is independent of the regulation of precursor cell proliferation. BMC Neurosci 7:77.

Poltyrev T, Yaka R (2013) Influence of abstinence and intervals between extinction trials on the expression of cocaine-conditioned place preference in adolescent rats. Psychopharmacology (Berl) 226:699-706.

Ribeiro Do Couto B, Aguilar MA, Lluch J, Rodriguez-Arias M, Minarro J (2009) Social experiences affect reinstatement of cocaine-induced place preference in mice. Psychopharmacology (Berl) 207:485-498.

Rogers JL, See RE (2007) Selective inactivation of the ventral hippocampus attenuates cue-induced and cocaine-primed reinstatement of drug-seeking in rats. Neurobiol Learn Mem 87:688-692.

Sartor GC, Aston-Jones G (2014) Post-retrieval extinction attenuates cocaine memories. Neuropsychopharmacology 39:1059-1065.
Siette J, Westbrook RF, Cotman C, Sidhu K, Zhu W, Sachdev P, Valenzuela MJ (2013) Age-specific effects of voluntary exercise on memory and the older brain. Biol Psychiatry 73:435442 .

Snyder JS, Ferrante SC, Cameron HA (2012) Late maturation of adult-born neurons in the temporal dentate gyrus. PLoS ONE 7:e48757.

Snyder JS, Soumier A, Brewer M, Pickel J, Cameron HA (2011) Adult hippocampal neurogenesis buffers stress responses and depressive behaviour. Nature 476:458-461.

Solinas M, Chauvet C, Thiriet N, El Rawas R, Jaber M (2008) Reversal of cocaine addiction by environmental enrichment. Proc Natl Acad Sci U S A 105:17145-17150.

Stone SS, Teixeira CM, Devito LM, Zaslavsky K, Josselyn SA, Lozano AM, Frankland PW (2011) Stimulation of entorhinal cortex promotes adult neurogenesis and facilitates spatial memory. J Neurosci 31:13469-13484.

Storey JD (2002) A direct approach to false discovery rates. J R Stat Soc Series B Stat Methodol 64:479-498.

Tashiro A, Makino H, Gage FH (2007) Experience-specific functional modification of the dentate gyrus through adult neurogenesis: a critical period during an immature stage. J Neurosci 27:3252-3259.

Taupin P (2007) BrdU immunohistochemistry for studying adult neurogenesis: paradigms, pitfalls, limitations, and validation. Brain Res Rev 53:198-214.

Torregrossa MM, Corlett PR, Taylor JR (2011) Aberrant learning and memory in addiction. Neurobiol Learn Mem 96:609623.

Tzschentke TM (2007) Measuring reward with the conditioned place preference (CPP) paradigm: update of the last decade. Addict Biol 12:227-462.

Vukovic J, Borlikova GG, Ruitenberg MJ, Robinson GJ, Sullivan RK, Walker TL, Bartlett PF (2013) Immature doublecortinpositive hippocampal neurons are important for learning but not for remembering. J Neurosci 33:6603-6613.

\section{SUPPORTING INFORMATION}

Additional Supporting Information may be found in the online version of this article at the publisher's web-site:

Figure S1 Effect of the TMZ treatment per brain hemisphere

Figure S2 Representative photographs of the areas quantified for c-Fos

Figure S3 Effect of TMZ on general health and behaviour Figure S4 Representative c-Fos staining in Sal- and CPP mice

Figure S5 C-Fos expression in low CPP-score VEH and TMZ mice

Figure S6 Extinction and reinstatement data expressed as seconds

Table S1 Relationship between hippocampal neurogenesis and the CPP-score

Table S2 Correlations among the brain areas analysed for c-Fos

Methods S1 Extended methods for Experiment 1

Methods S2 Extended methods for Experiment 2 